\title{
The classification of helium-rich hot subdwarfs ${ }^{\star}$
}

\author{
C.S. Jeffery ${ }^{1}$, J.S. Drilling ${ }^{2}$, P.M. Harrison ${ }^{3}$, U. Heber ${ }^{4}$, and S. Moehler ${ }^{4,5,6}$ \\ 1 Armagh Observatory, College Hill, Armagh BT61 9DG, Northern Ireland \\ 2 Dept. of Physics and Astronomy, Louisiana State University, Baton Rouge, Louisiana 70803, U.S.A. \\ 3 School of Physics \& Astronomy, University of St Andrews, Fife KY16 9SS, Scotland \\ 4 Dr.-Remeis Sternwarte, Universität Erlangen-Nürnberg, Bamberg D96049, Germany \\ 5 Landessternwarte, Königstuhl, D69117 Heidelberg, Germany \\ ${ }^{6}$ Space Telescope Science Institute, 3700 San Martin Drive, Baltimore, MD 21218, U.S.A.
}

Received January 16; accepted February 13, 1997

\begin{abstract}
Spectroscopy has been obtained of 23 subluminous stars, twenty of which had been classified as "heliumrich subdwarf B stars" from $10 \AA$ resolution spectra. From inspection of the He II, He I and Balmer lines, the sample clearly shows a wider range in $\mathrm{He} \mathrm{II} / \mathrm{He} \mathrm{I}$ and $\mathrm{He} / \mathrm{H}$ ratios than hitherto supposed for the class. We introduce a classification scheme for helium-rich hot subdwarfs based on $1.5 \AA$ spectra of high $\mathrm{S} / \mathrm{N}$, which can be extended to include all hot subdwarfs with spectra dominated by $\mathrm{H}$, He I or He II. The aim of this classification scheme is to establish reference standards which would be suitable for subsequent fine analysis.
\end{abstract}

Key words: stars: fundamental parameters — stars: subdwarfs

\section{Introduction}

A number of objects identified in the Palomar-Green survey of faint blue stars were classified sdO(D) (Green et al. 1986), having strong He I lines and weak or absent Balmer lines. Similar objects have been identified in other surveys of faint blue stars (Edinburgh-Cape, Hamburg-Schmidt) and are alternatively known as helium-rich subdwarf $\mathrm{B}$ stars (He-sdB) (Moehler et al. 1990). In an attempt to clarify the classifications currently in use, Drilling (1996) introduced the term sdB4 to denote these objects. A list complete to December 1995 was compiled by Jeffery et al. (1996).

Apart from spectroscopic identification the only previous work on these stars is a preliminary study which demonstrated the prototype PG1544+488 to be helium

\footnotetext{
Send offprint requests to: C.S. Jeffery

* Based on observations obtained with the Calar Alto $3.5 \mathrm{~m}$ and William Herschel telescopes.
}

enriched (Heber et al. 1988) and analyses of JL87 (Schulz et al. 1991), LS IV-14 116 (Viton et al. 1991) and LB 1766 (Kilkenny \& Busse 1992). These objects were shown to have similar $T_{\text {eff }}$ to the more numerous sdB stars, but slightly lower surface gravity and much higher helium abundances.

The existence of extremely helium-rich stars otherwise similar to the better known but extremely hydrogenrich subdwarf B stars suggests that they represent a vital link in the late stages of stellar evolution. A number of possibilities arise. Are they intrinsically the same objects as normal sdBs, but with their surface hydrogen mixed into helium-rich layers just below the surface? Are they more closely related to the hotter helium-rich sdOs lying close to the helium main- sequence and beyond the blue end of the horizontal branch? Or are they more closely related to the extreme helium stars, particularly the hottest high-gravity members of the class such as LSS 3184 (Drilling et al. 1997) and LS IV $+6^{\circ} 2$ (Jeffery 1996)?

In order to ascertain the nature of our targets more precisely, the authors have obtained optical spectroscopy and ultraviolet spectrophotometry of a significant sample. In this paper we examine the optical spectroscopy and review the classification of the targets. Refining the classification is a first step towards making more detailed atmospheric analyses of individual objects.

\section{Observations}

We have obtained spectra of 20 objects classified sdO(D) by Green et al. (1986) or He-sdB by Beers et al. (1992) and Heber et al. (1996), and spectra of a few objects classified $\operatorname{sdO}(\mathrm{A}), \operatorname{sdO}(\mathrm{B})$ and $\mathrm{sdO}(\mathrm{C})$ by Green et al. (1986). The latter were obtained to provide overlap between our sample and samples analyzed in other studies (e.g. Thejll et al. 1994; Saffer et al. 1994). For objects with $m_{V}<14$ spectra were obtained with the William Herschel telescope (WHT) and Utrecht échelle spectrograph (UES) in May 
Table 1. Optical spectroscopy of helium-rich subdwarf B stars. Magnitudes are primarily from Green et al. (1986)

\begin{tabular}{|c|c|c|c|}
\hline Object & $m_{B}$ & Instrument & Date \\
\hline PG0902+058 & 14.1 & $\mathrm{WHT}+\mathrm{UES}$ & 1995 May $6,7,8$ \\
\hline PG0902+058 & & $\mathrm{CA}$ & 1988 May 25 \\
\hline PG0909+276 & 10.7 & $\mathrm{WHT}+\mathrm{UES}$ & 1995 May $6,7,8$ \\
\hline PG0909+276 & & WHT+ISIS & 1996 May 30 \\
\hline PG0921+311 & 14.4 & WHT+ISIS & 1996 May 30 \\
\hline $\mathrm{HS} 1000+4704$ & 17.1 & WHT+ISIS & 1996 May 31 \\
\hline PG1127+019 & 13.0 & WHT+UES & 1995 May 6,7 \\
\hline PG1127+019 & & WHT+ISIS & 1996 May 31 \\
\hline PG1127+019 & & $\mathrm{CA}$ & 1988 May 26 \\
\hline PG1230+067 & 12.3 & WHT+UES & 1995 May $6,7,8$ \\
\hline TON107 & 16.3 & WHT+ISIS & 1996 May 30 \\
\hline PG1413+113 & 15.6 & $\mathrm{CA}$ & 1988 May 25 \\
\hline PG1415+492 & 14.4 & WHT+UES & 1995 May 7, 8 \\
\hline PG1415+492 & & $\mathrm{CA}$ & 1988 May 26 \\
\hline PG1437+727 & 13.6 & WHT+UES & 1995 May $7 \dagger$ \\
\hline PG1437+727 & & WHT+ISIS & 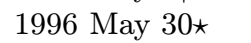 \\
\hline PG1441+407 & 15.5 & WHT+ISIS & 1996 May 31 \\
\hline PG1526+440 & 15.7 & WHT+ISIS & 1996 May 31 \\
\hline PG1544+488 & 12.0 & WHT+UES & 1995 May 7,8 \\
\hline PG1544+488 & & $\mathrm{CA}$ & 1988 May 26 \\
\hline PG1552+464 & 16.0 & WHT+ISIS & 1996 May 31 \\
\hline PG1554+408 & 15.9 & WHT+UES & 1995 May 9 \\
\hline PG1554+408 & & WHT+ISIS & 1996 May 31 \\
\hline PG1559+222 & 14.5 & WHT+UES & 1995 May 7, 8 \\
\hline PG1559+222 & & $\mathrm{CA}$ & 1988 May 26 \\
\hline PG1600+171 & 16.3 & WHT+ISIS & 1996 Jun. 1 \\
\hline PG1607+173 & 10.2 & WHT+ISIS & 1996 May 30 \\
\hline PG1615+413 & 16.4 & WHT+ISIS & 1996 Jun. 1 \\
\hline PG1629+466 & 14.0 & $\mathrm{CA}$ & 1988 May 27 \\
\hline PG1629+466 & & WHT+UES & 1995 May 9 \\
\hline PG1648+315 & 16.0 & WHT+ISIS & 1996 Jun. 1 \\
\hline PG1658+273 & 15.7 & WHT+ISIS & 1996 Jun. 1 \\
\hline PG1715+273 & 16.2 & WHT+ISIS & 1996 Jun. 1 \\
\hline HS1843+6343 & 16.1 & WHT+ISIS & 1996 May 30 \\
\hline LS IV-14 116 & 13.0 & WHT+ISIS & 1996 May 30 \\
\hline PG2128+096 & 14.3 & WHT+UES & 1995 May 9 \\
\hline PG2128+096 & & WHT+ISIS & 1996 May 30 \\
\hline PG2215+151 & 13.9 & WHT+ISIS & 1996 Jun. 1 \\
\hline
\end{tabular}

1995 covering the wavelength interval $3860-5025 \AA$ with a resolution $R=45000$. Fainter objects were observed with the WHT and intermediate dispersion spectrograph (ISIS) in the wavelength intervals $4240-4974 \AA$ and with a resolution of $R=2900$. Additional spectra were obtained with the $3.5 \mathrm{~m}$ telescope at Calar Alto (CA) in the wavelength interval $4181-5070 \AA$ with a resolution $R=2500$. Owing to a problem in the image-derotator, the $\mathrm{S} / \mathrm{N}$ of the UES observations in 1995 is poor, and to extract useful information the spectra have been binned to a resolution $R \sim 3000$. Further details are given in
Table 1. Moreover, with low $\mathrm{S} / \mathrm{N}$ in the UES spectra, and the substantial widths of important absorption lines relative to individual échelle orders in the UES spectra, there were rectification difficulties which could only be resolved where a low-resolution CA or ISIS spectrum was available. Thus, for example, the blue wing of the $\mathrm{H} \beta$ profile in our UES spectrum of PG0909+276 is incorrect (Fig. 5).

It was noted that spectra of three objects did not resemble subdwarf spectra at all.

PG1552+464: our spectrum shows strong and broad He I $\lambda 4471 \AA$, H $\beta$ slightly less strong, and weak He I 4713. $\mathrm{H} \gamma$ is just detectable. Our spectrum is too noisy to show any weaker features. This star would appear to be a DBA white dwarf.

PG1437+727: our UES spectrum is that of a G star. Our ISIS observations found a close pair, the fainter star being the subdwarf.

PG2128+096: our spectrum shows the weak Balmer lines, prominent $\mathrm{G}$ band and rich metal-line spectrum characteristic of a late-F or early-G star.

For the remainder it became evident that several objects show spectra substantially different from that expected from previous observations of the prototype PG1544+488. Although the majority show essentially no Balmer lines, weak He II and possibly a number of weak metal lines, in most cases He II $\lambda 4686 \AA$ is much stronger than in PG1544+488. Whilst it had been supposed that several objects would indicate effective temperatures around $\sim 30000 \mathrm{~K}$ and surface gravities between those of the extreme helium giants and the classical hydrogen-rich subdwarf B stars, the observed strength of He II $\lambda 4686 \AA$ is more often similar to that seen in sdOB and sdO stars. It is therefore necessary to review the classification of these stars.

To be properly useful, this review had to draw on a larger sample of spectral types than was provided by the observations described above. A number of $2.5 \AA$ spectra were obtained in a series of studies of hot subluminous stars at high galactic latitudes (Moehler et al. 1990) at the Calar Alto and the European Southern Observatories. These have been used to provide both comparison spectra and examples of hot subdwarf spectral types not already represented. These spectra include the well-known subdwarfs Feige 110 and $\mathrm{BD}+28^{\circ} 4211$. They also include PG1701+359 and PG2337+070 (Theissen et al. 1993), and PG1047+003, PG1559+048, PG1559+076, PHL 4, and PG1537-046, which have not previously been published. Since B-type subdwarf spectra are also similar to those of horizontal-branch B (HBB) stars, we have also examined a spectrum of PG1705+537 from the Moehler et al. (1990) sample. Echelle spectra of three helium stars obtained at ESO (LSS 5121: Heber et al. 1986), the WHT (LS IV+6² 2 ) and the AAT (HD 144941: Harrison \& Jeffery 1996) have also been used for comparison purposes. The resolution of 


\begin{tabular}{|c|c|c|c|c|}
\hline & $\mathrm{He}_{4471} \sim 0$ & & & $\mathrm{H} \gamma \sim 0$ \\
\hline $\mathrm{He}_{\mathrm{II}} 4686 \sim 0$ & $\begin{array}{l}\text { sdB1 } \\
\text { sdB }\end{array}$ & $\begin{array}{l}\text { sdB2 } \\
\text { sdB-O }\end{array}$ & $\begin{array}{l}\text { sdB3 } \\
\operatorname{sdO}(A)\end{array}$ & \begin{tabular}{|c|c|} 
sdB4 \\
sdO(D) He-sdB
\end{tabular} \\
\hline $\mathrm{HeII}_{4686} \sim \mathrm{He} \mathrm{I}_{4471}$ & & sdOB & $\operatorname{sdO}(\mathrm{B})$ & B2 \\
\hline \multirow[t]{2}{*}{$\mathrm{He}_{4471} \sim 0$} & \multicolumn{2}{|c|}{ sdO1 } & \multicolumn{2}{|l|}{$\operatorname{sdO}(\mathrm{C})$} \\
\hline & \multicolumn{2}{|c|}{$\mathrm{He} \mathrm{II}_{4686}<\mathrm{H} \gamma$} & \multicolumn{2}{|c|}{$\mathrm{He} \mathrm{II}_{4686} \gtrsim \mathrm{H} \gamma$} \\
\hline
\end{tabular}

Fig. 1. Schematic of classifications for $10 \AA$ resolution spectra of hot subdwarfs. The boxes are labelled by Drilling (1996) nomenclature in bold type and their equivalents as described in the text

the échelle spectra was degraded to match the remainder of the sample.

\section{Classification of $10 \AA ̊$ spectra}

Drilling (1996) discussed the classification of the spectra of hot subdwarfs of 1.5 to $10 \AA$ resolution and has identified 8 distinct classes, all of which had been previously defined in the literature. The spectra of ALL hot subdwarfs classified to date fit into one of these 8 classes which can be arranged in a two-parameter sequence of He I/He II versus $\mathrm{He} / \mathrm{H}$. Some duplicity of nomenclature exists, which Drilling sought to rationalise with a provisional scheme which is amplified here.

sdB (smallest He II/He I). "Stars with colours corresponding to those of a B star and in which the Balmer lines are abnormally broad for the colour as compared with Pop. I main-sequence stars. Some, but not all, subdwarf B stars have He I lines that are weak for their colour" (Sargent \& Searle 1968).

- sdB1. "Higher-gravity Balmer absorption over a large colour range, no He I 4471" (Green et al. sdB).

- sdB2. "sdB stars with a suggestion of HeI" (Green et al. sdB-O).

- sdB3. "dominant Balmer absorption along with pronounced He I 4471 and often He I 4026" (Green et al. $\operatorname{sdO}(\mathrm{A}))$.

- sdB4. "pure He I absorption spectra, characterized by the weakness or absence of hydrogen Balmer lines and He II 4686 while showing the singlet He I 4388 about equal in strength to the triplet $\mathrm{He}$ I 4471" (Green et al. sdO(D), Moehler et al. He-sdB). It is significant that this is the same definition as that given for extreme helium stars and binaries (Drilling 1986).

Closer examination suggests that there is little to distinguish classes sdB2 and sdB3 quantitatively. In terms of our classification lines, both classes fulfil the criterion that He I 4471 is present but weaker than $\mathrm{H} \gamma$.
sdOB (intermediate He II/He I)

- sdOB1. "strong broad Balmer lines accompanied by weak He I 4471 and He II 4686 absorption" (Moehler et al. sdOB)

- sdOB2. "dominated by HeI and HeII lines and generally showing hydrogen Balmer absorption" (Green et al. $\mathrm{sdO}(\mathrm{B}))$.

sdO (largest He II/He I)

- sdO1. "a star in which He II 4686 is seen, and which has, relative to main sequence stars, strong Balmer lines for the colour" (Sargent \& Searle sdO, not recognized by Green et al.; Moehler et al. sdO)

- sdO2. "dominated by He II absorption. Only a trace of He I 4471 may be present, and some Balmer absorption may be blended with He II Brackett lines" (Green et al. $\operatorname{sdO}(\mathrm{C})$, Moehler et al. He-sdO).

The two-dimensional sequence is illustrated schematically in Fig. 1. It should be noted that the Green et al. (1986) classifications refer to $10 \AA$ resolution spectra. It should also be noted that this spectral classification system remains incomplete after more than a decade of use. Standard stars observed at a specified resolution and $\mathrm{S} / \mathrm{N}$ have still to be established. In the following, we shall adopt the Drilling notation to refer to $10 \AA$ classifications.

\section{The need for a $1.5 \AA$ classification}

It has been noted that in many cases, our sample of $1.5 \AA$ spectra of sdB4 stars shows He II $\lambda 4686 \AA$ stronger than was expected from the definition of the class given by Green et al. (1986). As an extreme case, PG1600+171 and PG1441+407 show He II $\lambda 4686 \AA$ stronger than HeII $\lambda 4471 \AA$. At the other extreme, only two objects, PG1544+488 and PG1415+492, show He II $\lambda 4686 \AA$ weaker than He II $\lambda 4713 \AA$.

Since the $10 \AA$ classification spectra were obtained at lower resolution and signal-to-noise than the present observations, the reasons for the wide spread in apparent He II/He I ratios for a group of stars in the same $10 \AA$ class 
Table 2. Classification of "helium-rich" subdwarfs

$\begin{array}{llll}\text { Object } & \begin{array}{l}\text { Green } \\ \text { et al. }\end{array} & \begin{array}{l}\text { Moehler } \\ \text { et al. }\end{array} & \text { Us } \\ \text { PG0902+058 } & \text { sdO(D) } & & \text { sdO6:He3 } \\ \text { PG0909+276 } & \text { sdO(A) } & & \text { sdO7:He1 } \\ \text { PG0921+311 } & \text { sdO(B) } & & \text { sdO6:He3 } \\ \text { HS1000+4704 } & & \text { sdO6:He4 } \\ \text { PG1127+019 } & \text { sdO(D) } & \text { sdO6:He3 } \\ \text { PG1230+067 } & \text { sdO(B) } & \text { sdO7:He2 } \\ \text { TON107 } & \text { sdO(D) } & \text { sdO6:He4 } \\ \text { PG1413+113 } & \text { sdO(D) } & \text { sdO9:He4 } \\ \text { PG1415+492 } & \text { sdO(D) } & \text { sdO7?:He3† } \\ \text { PG1437+727 } & \text { sdO(D) } & \text { sdO5:He4 } \\ \text { PG1441+407 } & \text { sdO(D) } & \text { sdO7:He2 } \\ \text { PG1526+440 } & \text { sdO(D) } & \text { sdO9:He4 } \\ \text { PG1544+488 } & \text { sdO(D) } & \text { He-sdB } & \text { DBA } \\ \text { PG1552+464 } & \text { sdO(D) } & \text { sdO7:He4 } \\ \text { PG1554+408 } & \text { sdO(D) } & \text { sdO7:He3 } \\ \text { PG1559+222 } & \text { sdO(D) } & \text { sdO5:He4 } \\ \text { PG1600+171 } & \text { sdO(D) } & \text { sdB:He2 } \\ \text { PG1607+173 } & \text { sdO(A) } & \text { sdO8:He3 } \\ \text { PG1615+413 } & \text { sdO(D) } & \text { sdO8:He3 } \\ \text { PG1629+466 } & \text { sdO(D) } & \text { sdO8:He2 } \\ \text { PG1648+315 } & \text { sdO(D) } & \text { sdO7:He4 } \\ \text { PG1658+273 } & \text { sdO(D) } & \text { sdO7:He3 } \\ \text { PG1715+273 } & \text { sdO(D) } & \text { sdO8:He4 } \\ \text { HS1843+6343 } & & \text { sdO7:He1 } \\ \text { LS IV-14 116 } & & \text { sdO6:He4 } \\ \text { PG2215+151 } & \text { sdO(C) } & \end{array}$

$\dagger 4686 / 4713$ not measured.

can be appreciated. This would have been compounded by random errors introduced when classifying individual spectra. However, it is a fundamental principle of the classification process that standard spectra obtained at one resolution cannot be used to classify spectra obtained at a higher resolution. The MK process allows for the development of a sophisticated and autonomous classification system using, initially, line ratios and, ultimately, the entire spectrum. Since it can be refined to allow great precision in the description of stellar spectra and to select candidates for detailed study (Morgan 1984), it is both necessary and desirable to set up a system of standard classification spectra for high $\mathrm{S} / \mathrm{N}$ spectra of $1.5 \AA$ resolution. We have shown that this is eminently true from the point of view of our sample. In the remainder of this paper a suitable system is introduced.

\section{Classification of $1.5 \AA$ spectra}

\subsection{Criteria}

A classification system for spectra of hot subdwarfs obtained in the blue-violet at $1.5 \AA$ resolution or better and with $\mathrm{S} / \mathrm{N}>10$ is described. This system is an extension of that discussed by Drilling (1996) and is based on the observation that amongst hot subdwarfs there is a continuum of spectral types in three dimensions, being

(i) the ratio of He II lines to He I lines,

(ii) the ratio of Balmer lines to He I and/or He II lines and

(iii) the ratio of either He I triplets to singlets (e.g. 4471 to $4388 \AA$ ) or He II $\lambda 4686 \AA$ to He II Pickering series members.

These ratios correspond roughly to effective temperature, $\mathrm{H} / \mathrm{He}$ abundance ratio and density, but do not depend on any external calibrators or assumptions. The key lines for the $\mathrm{He} \mathrm{I} / \mathrm{He} \mathrm{II}$ and $\mathrm{H} / \mathrm{He}$ dimensions are $\mathrm{H} \gamma$, He I $\lambda 4713 \AA, H e$ I $\lambda 4471 \AA, H_{\text {I }} \lambda 4388 \AA$, He II $\lambda 4686 \AA$, and He II $\lambda 4543 \AA$. The criteria are best illustrated in a block diagram (Fig. 2).

At the low-temperature end of the scale, He II $\lambda 4686 \AA$ is undetectable, at the high-temperature end He I $\lambda 4471 \AA$ is undetectable. In between, the relative strengths of He I $\lambda 4713 \AA$, He I $\lambda 4471 \AA$ and He II $\lambda 4686 \AA$ establish a seven-point scale. In the $\mathrm{H} / \mathrm{He}$ dimension, the relative strengths of He I $\lambda 4471 \AA$ and $\mathrm{H} \gamma$ or of He II $\lambda 4543 \AA$ and $\mathrm{H} \gamma$ provide a four-point scale. If neither He I nor He II lines are seen but high-gravity Balmer lines are present, there is no spectral measure of effective temperature. Neglecting, for the present, the density dimension, these scales define a rectangular grid of 29 classification boxes.

It is clear that the present sample of "sdB4" stars can be arranged in extensive sequences in both temperature and $\mathrm{H} / \mathrm{He}$ directions, and may be found in eleven of these boxes. Some of these are illustrated in Figs. 3 and 4. A limited sequence in surface gravity is illustrated by a comparison of PG1544+488 and PG1415+492 with spectra of the extreme helium stars LS IV $+6^{\circ} 2$ and LSS 5121 (Fig. 5). A spread in gravity of 1.0 dex amongst He-rich sdO stars has already been established by Husfeld et al. (1989) and Dreizler (1993). However, the current sample contains insufficient members to quantify any general spectral discriminant.

It is also apparent from our $1.5 \AA$ resolution spectra that many hot subdwarfs show a rich spectrum of metal absorption lines. Whilst obvious examples include PG1607+173 with a metal-line spectrum typical of earlyB helium giants (cf. BD-94395, Jeffery \& Heber 1992), lines from more highly-ionized species can be also identified in spectra of the hottest objects including, for example, C IV $\lambda 4658 \AA$ and N v $\lambda 4604,4620 \AA$ in $\mathrm{BD}+28^{\circ} 4211$. However there is such diversity that the metal lines do not provide good primary classification criteria.

\subsection{Nomenclature}

The derivation of a useful nomenclature is complicated by the need to avoid conflict with established practice, but is recognizable in terms of familiar conventions. 


\begin{tabular}{|c|c|c|c|c|c|}
\hline & $\mathrm{He}_{4471}=0$ & $\mathrm{He}_{4471} \ll \mathrm{H} \gamma$ & $\mathrm{He}_{4471}=\mathrm{H} \gamma$ & $\mathrm{He}_{4471}>\mathrm{H} \gamma$ & $\mathrm{H} \gamma=0$ \\
\hline $\mathrm{He} \mathrm{II}_{4686} \sim 0$ & \multirow[t]{7}{*}{ sdB:He0 } & sdB:He1 & sdB:He2 & sdB:He3 & \\
\hline $\mathrm{He} \mathrm{II}_{4686}<\mathrm{He}_{4713}$ & & & & & sdO9:He4 \\
\hline $\mathrm{He} \mathrm{II}_{4686} \sim \mathrm{He}_{4713}$ & & & sdO8:He2 & sdO8:He3 & sdO8:He4 \\
\hline $\begin{array}{l}\mathrm{He} \mathrm{II}_{4686}>\mathrm{He} \mathrm{I}_{4713} \\
\mathrm{He} \mathrm{II}_{4686}<\mathrm{He} \mathrm{I}_{4471}\end{array}$ & & sdO7:He1 & sdO7:He2 & sdO7:He3 & sdO7:He4 \\
\hline $\mathrm{He} \mathrm{II}_{4686} \sim \mathrm{He} \mathrm{I}_{4471}$ & & sdO6:He1 & sdO6:He2 & sdO6:He3 & sdO6:He4 \\
\hline $\mathrm{He} \mathrm{II}_{4686}>\mathrm{He} \mathrm{I}_{4471}$ & & & sdO5:He2 & & sdO5:He4 \\
\hline \multirow[t]{2}{*}{$\mathrm{He}_{4471} \sim 0$} & & sdO4:He1 & sdO4:He2 & sdO4:He3 & sdO4:He4 \\
\hline & $\mathrm{He} \mathrm{II}_{4686}=0$ & $\mathrm{He} \mathrm{II}_{4543} \sim 0$ & $\mathrm{He} \mathrm{II}_{4543} \ll \mathrm{H} \gamma$ & $\mathrm{He} \mathrm{II}_{4543}<\mathrm{H} \gamma$ & $\mathrm{He} \mathrm{II}_{4543} \sim \mathrm{H} \gamma$ \\
\hline
\end{tabular}

Fig. 2. Schematic of $1.5 \AA$ line-ratio classification of hot subwarfs. Only classes currently identifed from observation are labelled

The prefix "sd" is a natural designation for subdwarf in current usage, which it is appropriate to preserve.

The classes "sdO" and "sdB" have their antecedents in the Harvard system for normal stars, whilst the hybrid "sdOB" is unique to the subdwarfs, indicating the presence of both He II and He I. It is the latter which causes the greatest difficulty, since the use of other letter and number combinations (e.g. OA, OC, OD, O1, O2, OB1, OB2, etc.) have not always followed self-evident sequences. The preferred parallel in the MK system is the sequence of subclasses "B0, O9, .., O5, O4" ${ }^{1}$. In our scheme we propose to drop the classification sdOB (which refers to a $\mathrm{He}$ II/He I ratio) and replace it with a scale running from sdB (implying no He II) through sdO9, sdO8, ... as required. Thus a true helium-rich sdB star would show no trace of He II, whilst a helium-rich star with no trace of He I would be classified sdO4. It is stressed that there is no one-to-one correspondence between the current sdO subtypes and the familiar MK O subtypes. It should also be remembered that, for example, an sdO4 subdwarf is much hotter than an O4V main-sequence star. Subdwarfs which show no helium lines cannot be further subclassified and would be labelled sdB:He0.

True helium-rich B stars (only He I and weak H) with high surface gravities include the helium stars V652 Her,

\footnotetext{
1 There are isolated cases of subdwarf classifications based on MK spectral types. HD49798 was designated sdO6 by Stickland \& Lloyd (1994) following Thackeray's (1970) description of the spectrum as O6. Such cases are sufficiently rare as to be unlikely to cause conflict.
}

LSS 3184 and HD 144941, but with surface gravities close to and lower than main-sequence values, these are not genuinely subluminous.

Regarding labelling the $\mathrm{H} / \mathrm{He}$ ratio, there is a choice between a numerical or alphabetic scale. Previous practice (e.g. Green et al.) suggests a set of alphabetic classes, "A" (hydrogen dominated) to "D" (helium dominated) via "B" and "C", whilst white dwarf classifications (Sion 1996) adopt "A" (pure H spectrum) to "B" (He I) or "O" (He II) via combinations such as "BA, OA" or "OB". The latter scheme is two-dimensional and insufficiently precise for our purposes. Moreover, in white dwarf (AOB) space, the "DA" white dwarfs occupy the same topological position as the "sdB" stars in hot subdwarf space! It is more convenient to provide a numerical scale running from "He1" (some He present) through "He4" (no hydrogen lines). The class "He0" only applies to the Hrich sdB stars referred to above. Although this numerical scale can be mapped exactly onto a concise alphabetic scale "A" through "D" similar to one already introduced (Green et al. 1986), the historical use of A and B to indicate $\mathrm{H}$ and $\mathrm{He} \mathrm{I}$ dominated spectra leads to ambiguity. This nomenclature is best understood in terms of the classification criteria by referring to Fig. 2 .

\subsection{Classifications}

We have defined a set of classification criteria for heliumlined hot subdwarfs which can be applied to $1.5 \AA$ resolution spectra of high $\mathrm{S} / \mathrm{N}$. We have proposed a classification 


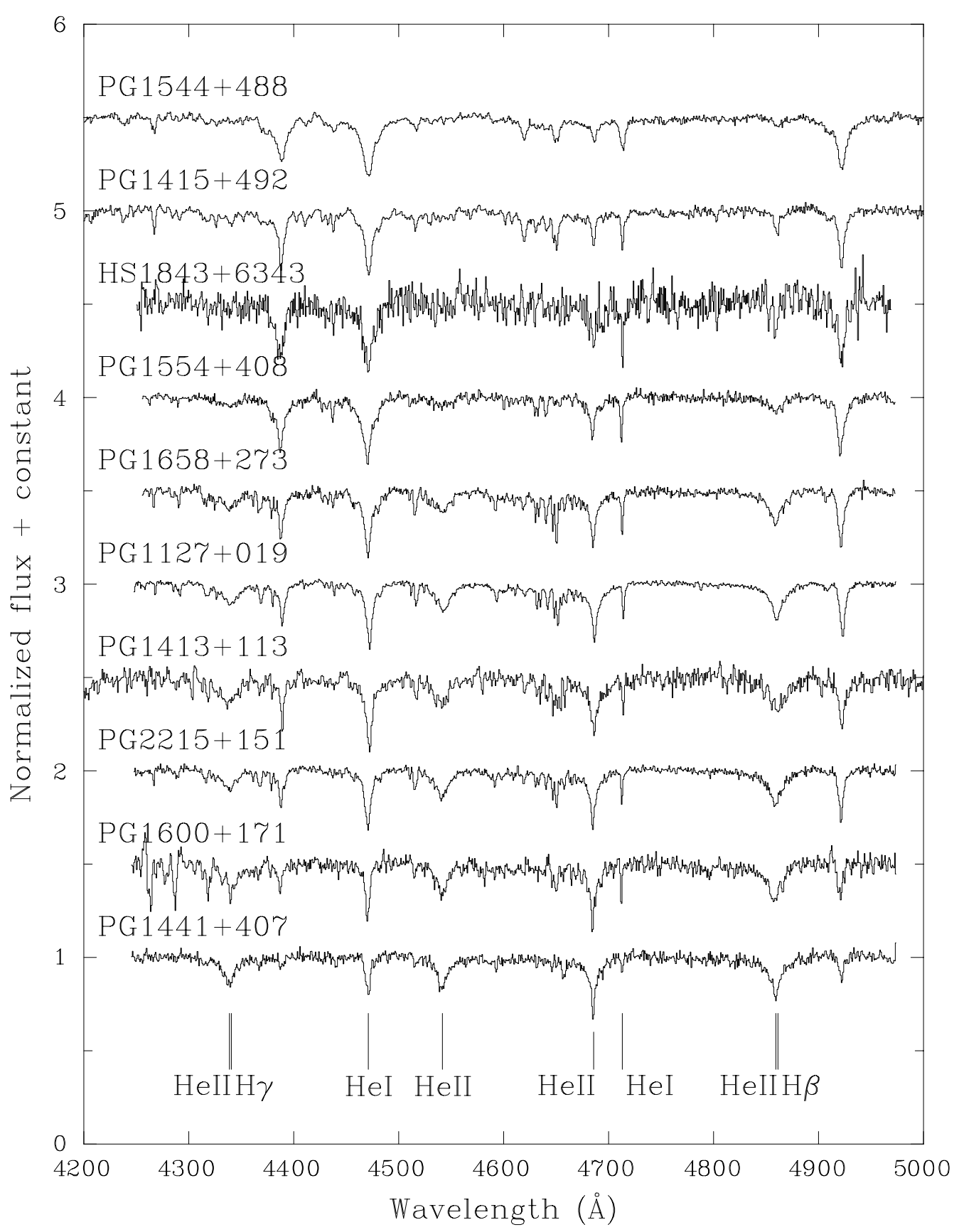

Fig. 3. Comparison of the spectra of helium-rich subluminous stars in which hydrogen lines are not detected. The objects are arranged in sequence of increasing He II/He I ratio (top-to-bottom). The positions of the classification lines are indicated. Lines at the positions of $\mathrm{H} \beta$ and $\mathrm{H} \gamma$ are inferred to be dominated by the Brackett series of He II by comparison with the intervening series member, He II $\lambda 4543 \AA$

nomenclature which may be applied to the system based on these criteria.

These have been applied to our sample of helium-rich hot subdwarfs, resulting in the classifications given in Table 2. For example, PG1715+273 shows HeII $\lambda 4686 \AA>$ He I $\lambda 4713 \AA, \quad$ He II $\lambda 4686 \AA<$ He I $\lambda 4471 \AA$ and He I $\lambda 4471 \AA>\mathrm{H} \gamma$, and so is classified sdO7:He3.
Further spectral classes have been identified from the sample of Moehler et al. (1990). All classes identified are illustrated in Fig. 6. As the classification of subdwarfs under this scheme proceeds, standards will become better established for those classes already identified, and identified for those classes not yet established. 


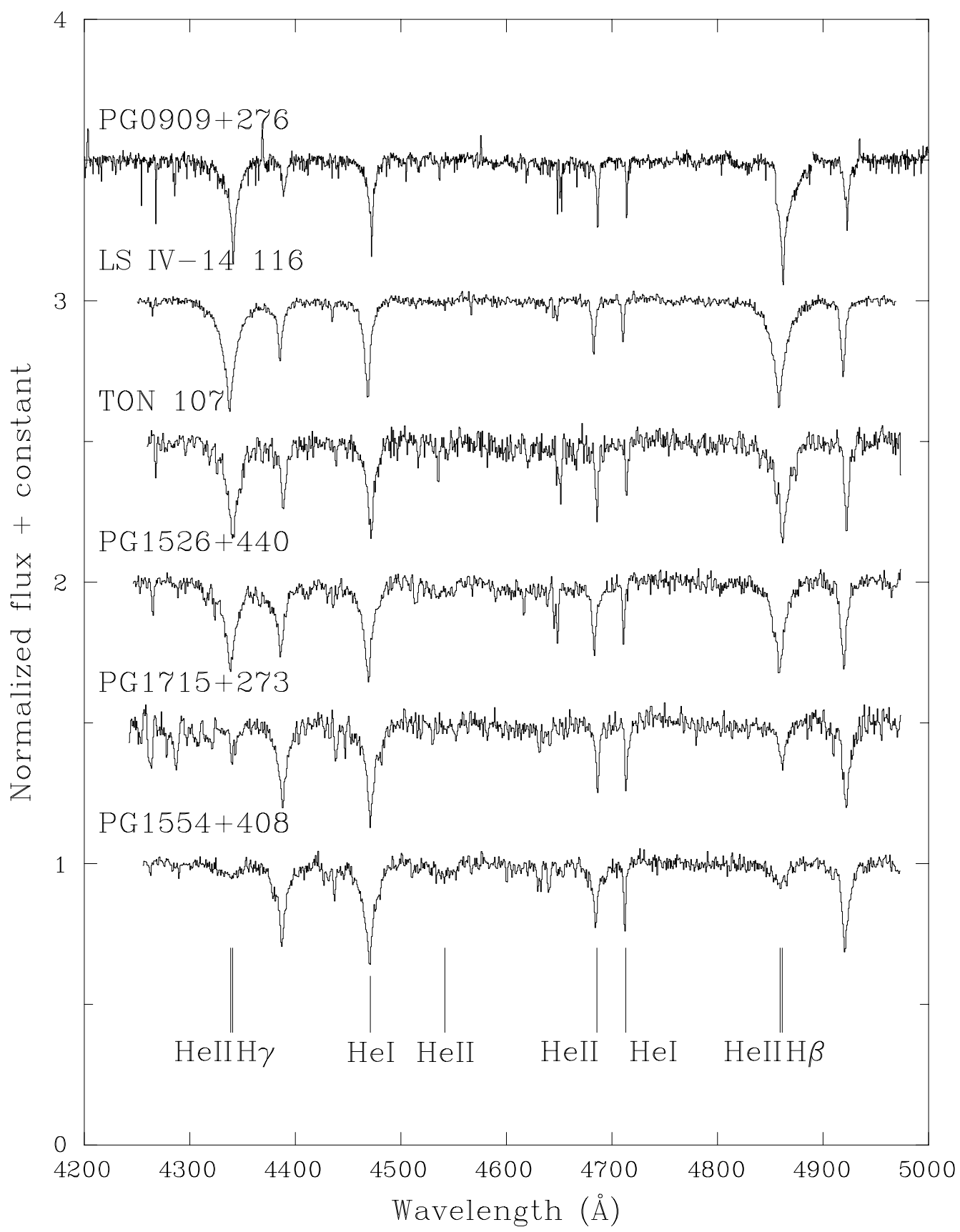

Fig. 4. Comparison of the spectra of helium-rich subluminous stars with a roughy equal HeII/He I ratio. The objects are arranged in sequence of increasing $\mathrm{He} / \mathrm{H}$ ratio (top-to-bottom). The positions of the classification lines are indicated

\section{Future work}

The proposed classification of $1.5 \AA$ spectra of hot subdwarfs has obvious merits for selecting targets for more detailed study, for providing a precise nomenclature for hot subluminous stars and for making statistical studies of the hot subdwarf population. A number of deficiencies still need to be addressed.

- A large number of subluminous stars with a non- sdB4 designation will be included to check the proposed classifications and to provide standards for currently empty boxes.

- The density dimension will be calibrated, including a spectral definition of the difference between $\operatorname{sdB}$ and HBB stars.

- The behaviour of the metal absorption lines must be quantified. Are there metal-poor and metal-rich subdwarfs, or C-rich and N-rich sequences?

- Spectra showing Balmer lines and no helium lines are degenerate - all are classified sdB:He0. Are there secondary indicators of effective temperature? 


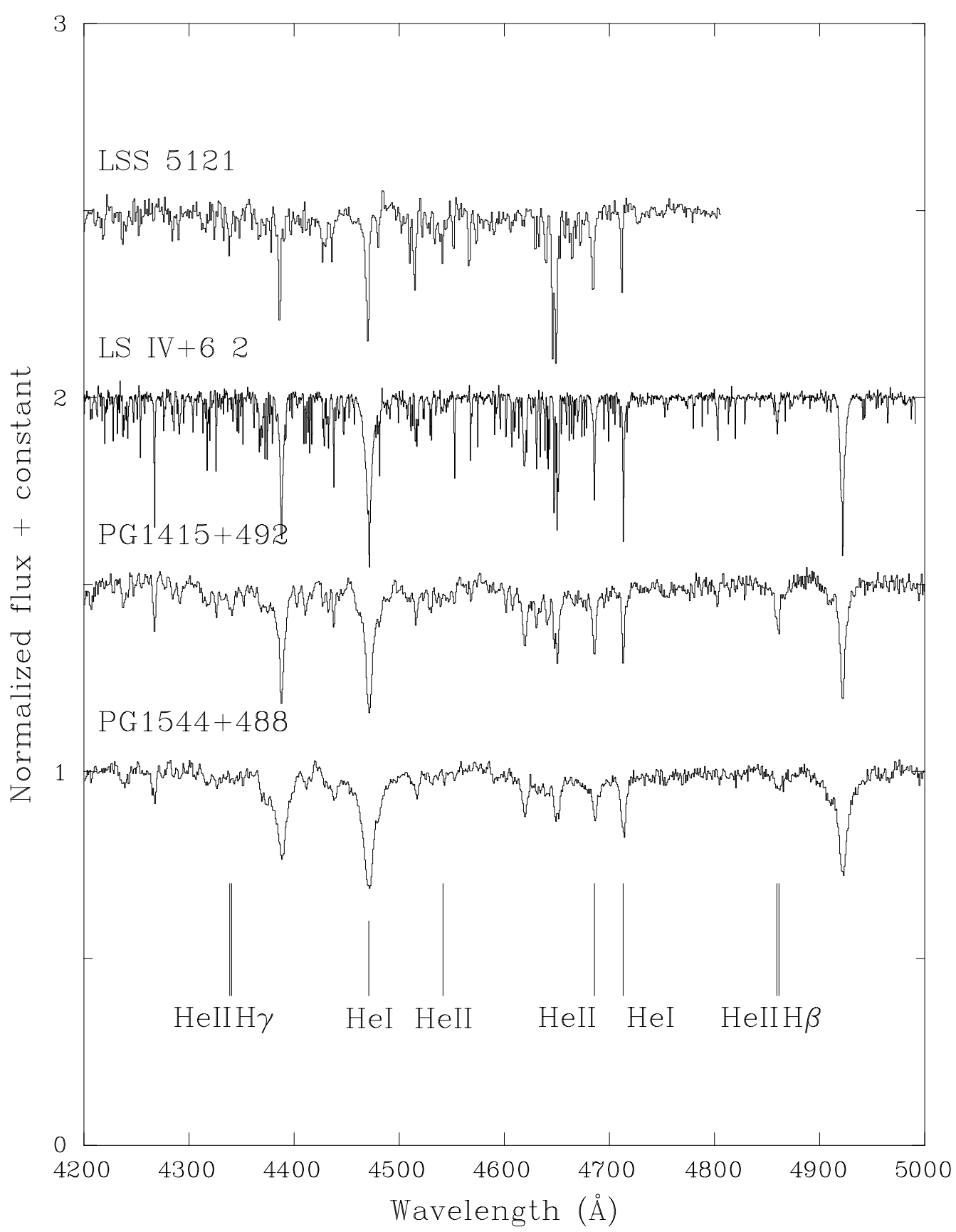

Fig. 5. Comparison of the spectra of helium-rich subluminous stars in which hydrogen-lines are very weak or not detected and He II $\lambda 4686 \AA$ is weaker than He I $\lambda 4713 \AA$. The objects are arranged in sequence of increasing He I line-width (top-to-bottom). The positions of the classification lines are indicated

- For spectra dominated by He II, the scheme becomes degenerate as He I $\lambda 4471 \AA$ disappears. An extension to higher temperatures will require alternative diagnostics (cf. Werner 1992).

It should be emphasized that the proposed scheme does not replace the $10 \AA$ classifications (e.g. Green et al. 1986). There will be circumstances such as studies of subdwarfs in distant clusters or further survey work where one will be forced to work at the lower resolution.

\section{References}

Beers T.C., Preston G.W., Schectman S.A., Doinidis S.P., Griffin K.E., 1992, AJ 103, 267

Dreizler S., 1993, A\&A 273, 212

Drilling J.S., 1986, "Hydrogen-Deficient Stars and Related Objects", Hunger K., Schönberner S., Rao N.K. (eds.) p. 9

Drilling J.S., 1996, "Hydrogen-Deficient Stars", Jeffery C.S., Heber U. (eds.), ASP Conf. Ser. 96, 461

Drilling J.S., Jeffery C.S., Heber U., 1997, A\&A (submitted) 


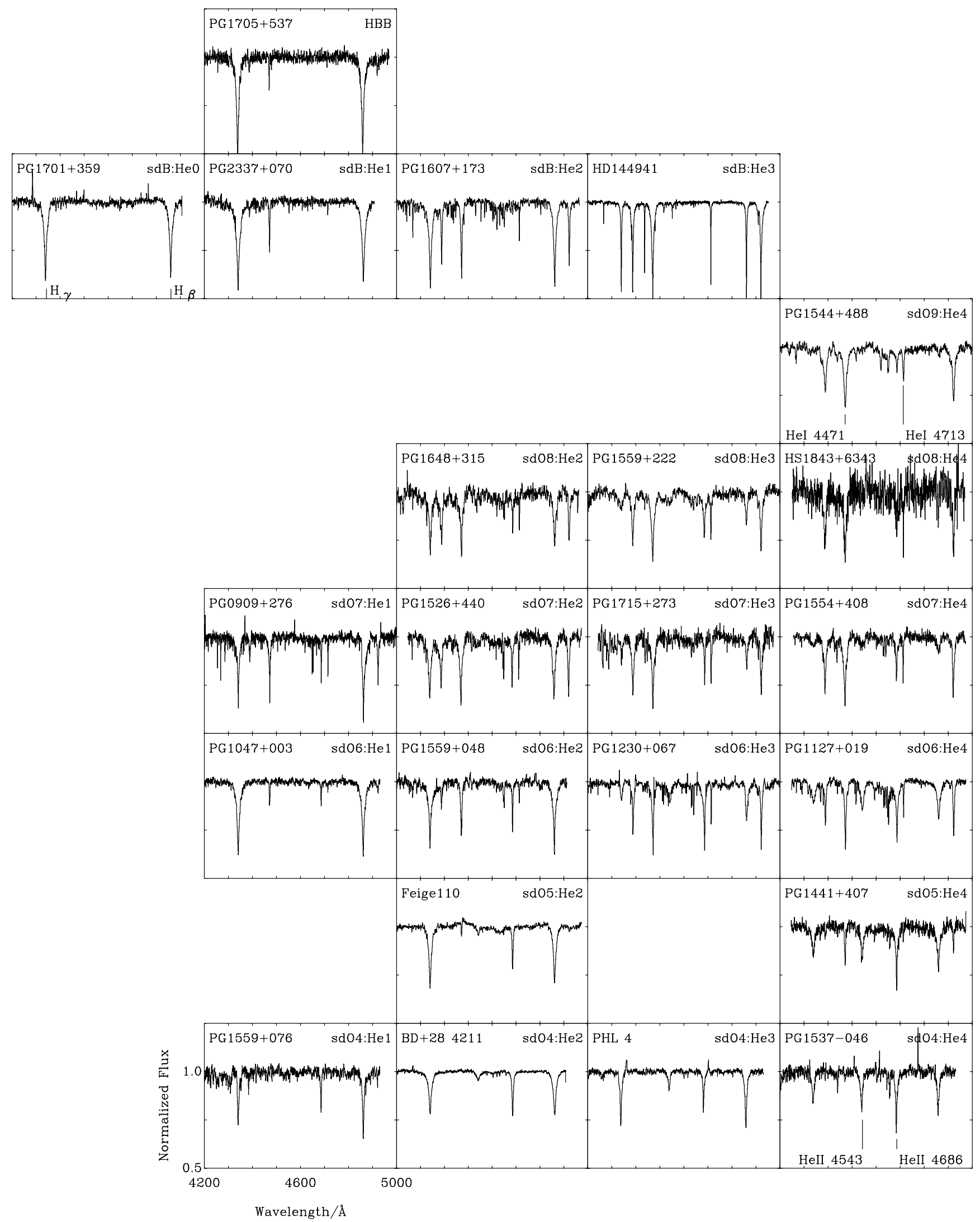

Fig. 6. An illustration of spectral classifications in the $\mathrm{H} / \mathrm{He}$ and $\mathrm{He} \mathrm{I} / \mathrm{He}$ II dimensions for selected hot subdwarfs. Examples of spectral types not represented in our sample of helium-rich subdwarfs are taken from Moehler et al. (1990) 
Green R.F., Schmidt M., Liebert J., 1986, ApJS 61, 305

Harrison P.M., Jeffery C.S., 1997, A\&A 323, 177

Heber U., Jonas G., Drilling J.S., 1986, in "Hydrogendeficient stars and related systems", IAU Coll. 87, Hunger K., Schönberner D., Rao N.K. (eds.). Reidel, Dordrecht, Holland

Heber U., Dreizler S., de Boer K.S., Moehler S., Richtler T., 1988, Astron. Ges. Abs. Ser. 1, 16

Heber U., Dreizler S., Werner K., Engels D., Hagen H.-J., 1996, "Hydrogen-Deficient Stars", Jeffery C.S., Heber U. (eds.), ASP Conf. Ser. 96, 241

Husfeld D., Butler K., Heber U., Drilling J.S., 1989, A\&A 222, 150

Jeffery C.S., 1996, "Hydrogen-Deficient Stars", Jeffery C.S., Heber U., ASP Conf. Ser. 96, 152

Jeffery C.S. Heber U., 1992, A\&A 260, 133

Jeffery C.S., Heber U., Hill P.W., et al., 1996, "HydrogenDeficicient Stars", Jeffery C.S., Heber U., ASP Conf. Ser. 96,471

Kilkenny D., Busse J., 1992, MNRAS 258, 57
Moehler S., Richtler T., de Boer K.S., Dettmar R.J., Heber U., 1990, A\&AS 86, 53

Morgan W.W., 1984, "The MK Process and Stellar Classification", Garrison R.F. (ed.), David Dunlap Observatory, p. 18

Saffer R.A., Bergeron P., Koester D., Liebert J., 1994, ApJ 432,351

Sargent W.L.W., Searle L., 1968, ApJ 152, 443

Schulz H., Heber U., Wegner G., 1991, PASP 103, 435

Sion E.M., 1996, "Hydrogen-Deficient Stars", Jeffery C.S., Heber U. (eds.), ASP Conf. Ser. 96, 223

Stickland D.J., Lloyd C., 1994, Observatory 114, 41

Thackeray A.D., 1970, MNRAS 150, 215

Theissen A., Moehler S., Heber U., de Boer K.S., 1993, A\&A 273,524

Thejll P., Bauer F., Saffer R., et al., 1994, ApJ 433, 819

Viton M., Deleuil M., Tobin W., Prévot L., Bouchet P., 1991, A\&A 242, 175

Werner K., 1992, "The Atmospheres of Early-Type Stars", Heber U., Jeffery C.S. (eds.). Springer-Verlag, p. 273 